EPSC Abstracts

Vol. 14, EPSC2020-250, 2020

https://doi.org/10.5194/epsc2020-250

Europlanet Science Congress 2020

(C) Author(s) 2020. This work is distributed under

the Creative Commons Attribution 4.0 License.

\title{
Planetary Sample Analysis Laboratory (SAL) at DLR
}

Jörn Helbert, Enrica Bonato, and Alessandro Maturilli

DLR, Institute for Planetary Research, Berlin, Germany (joern.helbert@dlr.de)

Introduction: Building on the available infrastructure and the long heritage in spectral studies of planetary (analog) materials DLR is creating a Sample Analysis Laboratory (SAL). The setup has started with the installation of a vis-IR-microscope at the Planetary Spectroscopy Laboratory in 2018. Full funding has been approved in December 2019.

SAL will add over the next 3 years capabilities for detailed mineralogical and geochemical characterization of material return by sample return missions in a clean room facility. The step-wise extension follows the successful development approach used for the Planetary Spectroscopy Laboratory (PSL) and Astrobiology Laboratories. The goal is to test and validate each extension step before planning the follow-up step. The first step is focused on analysing samples from asteroids missions like Hayabusa 2 mission, Osiris-REX and lunar sample return missions. SAL can later be extended to a full Sample Curation facility.

Global reconnaissance of planetary surface can only be obtained by remote sensing methods. Optical spectroscopy from UV to far-infrared is playing a key role to determine surface mineralogy, texture, weathering processes, volatile abundances etc. It is a very versatile technique, which will continue to be of importance for many years to come. Providing ground truth by in-situ measurements and ultimately sample return can significantly enhanced the scientific return of the global remote sensing data. This motivates the planned extension of PSL with a SAL by support of the Astrobiology Laboratories.

SAL will focus on spectroscopy on the microscopic scale and geochemical and geo-microbiological analysis methods to study elemental composition and isotopic ratios in addition to mineralogy to derive information on the formation and evolution of planetary surfaces, search for traces of organic materials or even traces of extinct or extant life and inclusions of water.

The DLR SAL will be operated as a community facility (much like PSL), supporting the larger German and European sample analysis community

Current facilities: PSL at DLR (http://s.dlr.de/2siu) is the only spectroscopic infrastructure in the world with the capability to measure emissivity of powder materials, in air or in vacuum, from low to very high temperatures, over an extended spectral range. Emissivity measurements are complimented by reflectance and transmittance measurements produced simultaneously with the same setup. It is the ground reference laboratory for the MERTIS thermal infrared spectral imager on the ESA BepiColombo mission. Members of the PSL group are team members of the MarsExpress, VenusExpress, MESSENGER and JAXA Hayabusa 2 missions. For the latter mission PSL has performed ground calibration measurements. In addition PSL has been used extensively in support 
of the ESA Rosetta mission. The samples analyzed at PSL ranged from rocks, minerals, to meteorites and Apollo lunar soil samples.

In a climate-controlled environment PSL operates currently two Fourier Transform Infrared Spectrometer (FTIR) vacuum spectrometers, equipped with internal and external chambers, to measure emittance, transmittance and reflectance of powdered or solid samples in the wavelength range from 0.3 to beyond 100 micron. Recently a Hyperion 2000 microscope has been added in preparation of the SAL setup.

The institute is also operating a Raman micro-spectrometer lab (http://s.dlr.de/e49q) with a spot size on the sample in focus of $<1.5 \mu \mathrm{m}$. The spectrometer is equipped with a cryostat serving as a planetary simulation chamber which permits simulation of environmental conditions on icy moons and planetary surfaces, namely pressure (10-6 hPa - $1000 \mathrm{hPa})$, atmospheric constituents, and temperature ( $4 \mathrm{~K}-500 \mathrm{~K})$. The samples, which are analyzed in the laboratory range from minerals, Martian analog materials, meteorites, biological samples (e.g. pigments, cell wall molecules, lichens, bacteria, archaea and other) to samples returned from the ISS (BIOMEX) and the asteroid Itokawa (Hayabusa sample).

All laboratory facilities undergo regular evaluations as part of the DLR quality management process. The evaluations address laboratory protocols, documentation, safety, data archival and staff training.

PSL is a community facility as part of the "Distribute Planetary Simulation Facility" in European Union funded EuroPlanet Research Infrastructure (http://www.europlanet-2020-ri.eu/). Through this program (and its predecessor) over the last 7 years more than 60 external scientists have obtained time to use the PSL facilities. PSL has setup all necessary protocols to support visiting scientist, help with sample preparation, and archive the obtained data.

Sample Analysis Laboratory: The near-term goal of the first step is the preparation to receive samples from the JAXA Hayabusa 2 and MMX missions, the Chinese Chang-E 5 and 6 missions as well as the NASA Osiris-REX mission. The current PSL and Raman facilities are operating in climatecontrolled rooms and follow well-established cleanliness standards. The SAL will be housed in two ISO 5 clean rooms. The cleanrooms are equipped with glove boxes to handle and prepare samples. All samples will be stored under dry nitrogen and can be transported between the instruments in dry nitrogen filled containers.

To characterize and analysis the returned samples the existing analytical capabilities are currently been extended. PSL was just upgraded with a vis-IR-microscope to extend spectral analysis to the sub-micron scale.

For the SAL this will be complemented by the following capabilities:

Based on current planning the first parts of SAL will be operational and ready for certification by end of 2021. Analysis of first Hayabusa 2 samples can start by beginning to mid of 2022.

Outlook: DLR has started establishing a Sample Analysis Laboratory. Following the approach of a distributed European sample analysis and curation facility as discussed in the preliminary recommendations of EuroCares (http://www.euro-cares.eu/) the facility at DLR could be expanded to a curation facility. The timeline for this extension will be based on the planning of sample return missions. The details will depend on the nature of the returned samples. Through the BIOMEX project a collaboration has been established with the Robert-Koch Institute (RKI) (http://www.rki.de) for question of samples that might pose a bio-hazard. RKI is operating BSL 4 facilities, which might be used as part of the DLR curation facilities. 